\title{
MHD mixed convection flow adjacent to a vertical plate with prescribed surface temperature
}

\begin{abstract}
The steady MHD mixed convection flow adjacent to a bounding surface immersed in an incompressible viscous fluid is considered. The governing system of partial differential equations is first transformed into a system of ordinary differential equations, before being solved numerically by a finite-difference scheme. The features of the flow and heat transfer characteristics for different values of the governing parameters are analyzed and discussed. Numerical results are obtained for the skin friction coefficient and the local Nusselt number as well as the velocity and temperature profiles. It is found that dual solutions exist for both assisting and opposing flows. The range of the mixed convection parameter for which the solution exists increases in the presence of a magnetic field.
\end{abstract}

Keyword: Boundary layer; Dual solutions; Heat transfer; Magnetohydrodynamic 